\title{
Gender Recognition in Informal and Formal Language Scenarios via Transfer Learning.
}

\author{
Daniel Escobar-Grisales ${ }^{1[0000-0002-3257-0134]}$, Juan Camilo \\ Vásquez-Correa ${ }^{1,2,3[0000-0003-4946-9232]}$, and Juan Rafael \\ Orozco-Arroyave ${ }^{1,2[0000-0002-8507-0782]}$ \\ 1 GITA Lab. Faculty of Engineering, University of Antioquia UdeA, Medellín, \\ Colombia. \\ 2 Pattern Recognition Lab. Friedrich-Alexander-Universität Erlangen-Nürnberg, \\ Germany \\ 3 Pratech Group, Medellín, Colombia. \\ \{daniel.esobar, jcamilo.vasquez, rafael.orozco\}@udea.edu.co
}

\begin{abstract}
The interest in demographic information retrieval based on text data has increased in the research community because applications have shown success in different sectors such as security, marketing, heathcare, and others. Recognition and identification of demographic traits such as gender, age, location, or personality based on text data can help to improve different marketing strategies. For instance it makes it possible to segment and to personalize offers, thus products and services are exposed to the group of greatest interest. This type of technology has been discussed widely in documents from social media. However, the methods have been poorly studied in data with a more formal structure, where there is no access to emoticons, mentions, and other linguistic phenomena that are only present in social media. This paper proposes the use of recurrent and convolutional neural networks, and a transfer learning strategy for gender recognition in documents that are written in informal and formal languages. Models are tested in two different databases consisting of Tweets and call-center conversations. Accuracies of up to $75 \%$ are achieved for both databases. The results also indicate that it is possible to transfer the knowledge from a system trained on a specific type of expressions or idioms such as those typically used in social media into a more formal type of text data, where the amount of data is more scarce and its structure is completely different.
\end{abstract}

Keywords: Demographic Information Retrieval · Gender Recognition · Transfer Learning · Recurrent Neural Networks · Convolutional Neural Networks.

\section{Introduction}

Demographic information retrieval consists in recognizing traits from a human being such as age, gender, personality, emotions, and others. Typically the main

\footnotetext{
* Supported by University of Antioquia.
} 
aim is to create a user profile based on unstructured data. The retrieval of such information has different applications in forensics, security, sales, marketing, health-care, and many other sectors [9]. In e-commerce scenarios, this type of information provides advantages to companies in competitive environments because it allows to segment customers in order to offer personalized products and services which strengths their marketing strategies [48]. Although most of the demographic factors are explicitly collected through the registration process, this approach could be limited given that most of potential customers in online stores are anonymous. The automatic recognition of demographic variables such as gender can help to overcome these limitations [5].

Text data from customers can be obtained via transliterations of voice recordings, chats, surveys, and social media. These text resources can be processed to automatically recognize the gender of the users. Different studies have applied Natural Language Processing (NLP) techniques for gender recognition in text data, mainly from social media posts. In [12 13 the authors used Term Frequency-Inverse Document Frequency (TF-IDF) to extract features from tweets in the PAN17 corpus [16, and reported accuracies for gender classification around $81 \%$. The authors in 3 used extracted features from TF-IDF as well as specific information only available in social media posts such as the frequency of female- and male-emojis. The authors reported an accuracy of $83.2 \%$ in the PAN17 [16] corpus for gender recognition. Although the high accuracy reported in the study, the methodology would not be accurate to model text data written in more formal scenarios such as customer reviews, product surveys, opinion posts, and customer service chats, which have a different structure compared to the texts that can be found in social media data. In other study [11, the authors proposed a system to classify the gender of the persons who wrote 100,000 posts from Weibo (Chinese social network similar to Tweeter). The system was based on a Word2Vec model, which achieved an accuracy of $62.9 \%$. The authors compared the performance of their model with human judgments, which accuracy was $60 \%$. This fact evidences that the problem of recognizing gender in written texts is very hard even for human readers. Wod2Vec models were also considered in 1 for gender recognition in the PAN17 corpus. The authors reported an accuracy of $69.5 \%$ for the Tweets in Spanish. There are some studies focused on gender classification using Deep Learning (DL) methods. However, when considering texts in Spanish, the number of studies is relatively small 911. In [10], the authors proposed a methodology based on Bidirectional Gated Recurrent Units (GRUs) and an attention mechanism for gender classification in the PAN17 corpus. The authors worked with a Word2Vec model as input for their DL architecture and reported accuracies of up to $75.3 \%$.

According to the reviewed literature, gender classification based on text data has been mainly explored in social media scenarios, where the language is informal and the documents do not follow a formal structure [6. These types of documents use a number of language variants, styles, and other content like emojis that help to accurately recognize different demographic information. There is a gap between models trained on formal and informal written language because 
a trained model with formal language data for a specific purpose will not achieve comparable results on an informal language scenario, or vice-versa [7. Due to this reason, it is important to validate trained models for gender recognition in both types of languages: formal and informal. In addition, the recognition of demographic variables such as gender are under-explored in documents with a more formal structure.

This paper proposes a methodology based on Recurrent Neural Networks (RNNs) and Convolutional Neural Networks (CNNs) for gender recognition in informal and formal language scenarios. First, the models are trained and tested in the PAN17 corpus, which is a traditional dataset for gender classification in Tweets. The models originally trained using the PAN17 corpus are re-trained using a transfer learning strategy with data from call-center conversations, which are structured in a more formal language. Accuracies of up to $75 \%$ are obtained, indicating that the proposed methodology is accurate for gender classification in documents written in formal and also in informal languages. Moreover, fine-tuned models using transfer learning show that despite the noise and lack of structure in documents written in informal language, they can be used to improve the accuracy of gender classification.

\section{Materials and Methods}

\section{$2.1 \quad$ Data}

PAN17: We are particularly working with the Spanish data of the corpus, namely PAN-CLEF 2017 [16. In this database, there are variants of Spanish from seven countries: Argentina, Chile, Colombia, Mexico, Peru, Spain and Venezuela. The training set is composed by texts from 600 subjects from each country (300 female). Since each subject has 100 Tweets, there is a total of 4200 subjects and 420000 Tweets in the dataset. The test set comprises data from 400 subjects from each country (200 female) for a total of 2800 subjects and 280000 Tweets. For the sake of comparison with previous studies, we kept the original train and test sets. The training set was randomly divided into $80 \%$ for training and $20 \%$ to optimize the hyper-parameters of the models (development set). All data distribution was performed subject independent to avoid subject specific bias and to guarantee a better generalization capability of the models.

Call Center Conversations: This corpus contains transliterations of conversations between customers and agents from a customer service center of a pension

administration company in Colombia. Texts are manually generated by a group of linguistic experts based on the audio signals from the customers. Similarly, the label of the gender is assigned based on the audio recordings processed by the linguists. Formal language is typically used by the customers when asking for a service, making a request, asking about certificates, and other questions about the service provided by the company. This database comprises 220 transliterations of different customers (110 female). The average number of words for each conversation is 602 , with a standard deviation of 554 . 


\subsection{Deep Learning Architectures for Gender Classification}

We consider two DL architectures in this case: an RNN with Bidirectional Long Short Term Memory (LSTM) cells, and a CNN with multiple temporal resolutions. These networks are trained with data from the PAN17 corpus. Then, a transfer learning strategy is applied to recognize gender from the call center conversations data.

Bidirectional Long Short Term Memory: The main idea of RNNs is to model a sequence of feature vectors based on the assumption that the output depends on the input features at the present time-step and on the output at the previous time-step. Conventional RNNs have a causal structure, i.e., the output at the present time step only contains information from the past. However, many applications require information from the future [15. Bidirectional RNNs are created to address such a requirement by combing a layer that processes the input sequence forward through time with an additional layer that moves backwards the input sequence. Traditional RNNs also exhibit a vanishing gradient problem, which appears when modeling long temporal sequences. LSTM layers were proposed to solve this vanishing gradient problem by the inclusion of a long-term memory to produce paths where the gradient can flow for long duration sequences such as sentences of a Tweet, or the ones that appear in a conversation with a call-center agent [17. We proposed the use of a Bidirectional LSTM (Bi-LSTM) network for our application. These architectures are widely used for different NLP tasks such as sentiment analysis in social media and product reviews 2 214/18]. A scheme of the considered architecture is shown in Fig. 1 .

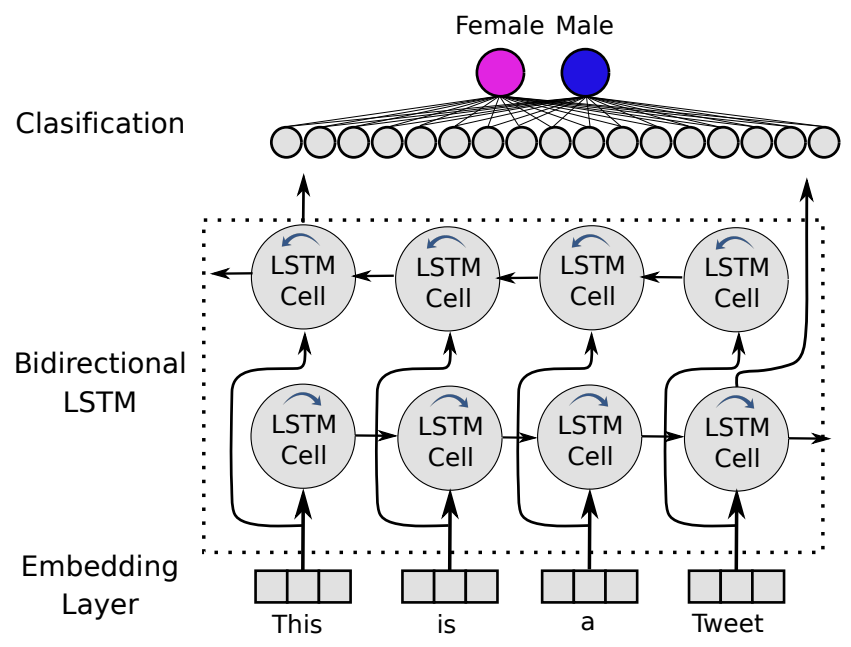

Fig. 1. Bi-LSTM architecture for gender classification in a Tweet. 
Words from the data are represented using a word-embedding layer. The input to the Bi-LSTM layer consists of $k d$-dimensional words-embedding vectors, where $k$ is the length of the sequence. The final decision about the gender of the subject is made at the output layer by using Softmax activation function.

Convolutional Neural Network (CNN): CNN-based architectures are designed to extract sentence representations by a composition of convolutional layers and a max-pooling operation over all resulting feature maps. We proposed the use of a parallel CNN architecture with different filter orders to exploit different temporal resolutions at the same time. Details of the architecture can be found in Fig. 2. The output from the word-embedding layer is convolved with filters of different orders $(n)$ and that correspond to different number of the $n$ in $n$-grams. The proposed CNN computes the convolution only in the temporal dimension. After convolution, a max-pooling operation is applied. Finally, a fully connected layer is used for classification using a Softmax activation function.

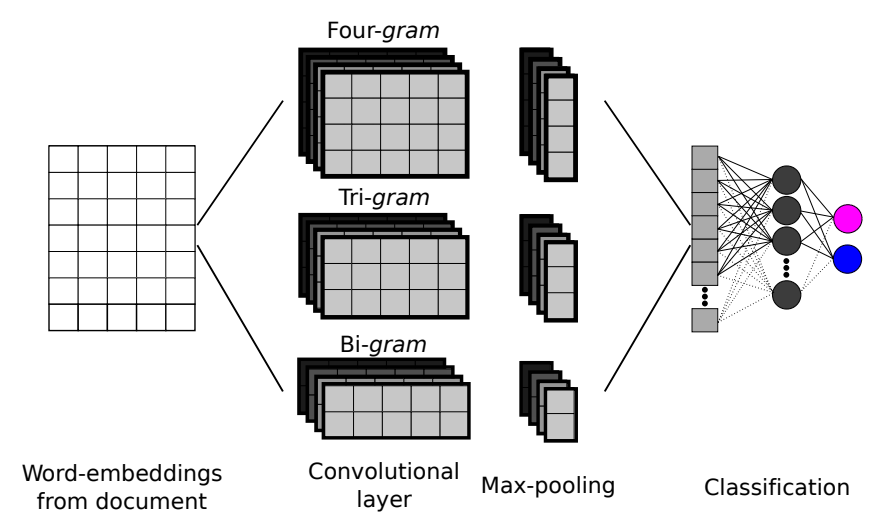

Fig. 2. CNN architecture for gender classification in a Tweet.

\subsection{Training}

The networks considered in this work are implemented in Tensorflow 2.0, and are trained with a sparse categorical cross-entropy loss function using an Adam optimizer. An early stopping strategy is used to stop training when validation loss does not improve after 10 epochs. The embedding dimension $d$ is set to 100 . The vocabulary size for the tokenizer is set to 5000 for the PAN17 corpus and 1500 for the call-center conversations. The difference between the two vocabulary sizes is given because the number of unique words present in the training sets of each database. Hyper-parameters are optimized upon the validation accuracy and the simplest model. 


\subsection{Transfer Learning}

We tested two approaches for the call-center conversations data: (1) training the network only using the data from the corresponding corpus, and (2) training the model via transfer learning by using a pre-trained model generated with the PAN17 corpus. For the transfer learning approach, the most accurate model for the PAN17 data is fine-tuned, but freezing the embedding layer in order to keep the tokenizer and a bigger vocabulary. Experiments without freezing the embedding layer were also performed but the results were not satisfactory. The motivation for using transfer learning is to test whether the knowledge learned by a model trained with text data in informal language is useful to improve gender classification systems based on text with formal language, since it is generally common to collect large amounts of data with informal structure through social media, but it is difficult to collect written documents with a formal structure.

\section{Experiment and Results}

Two experiments are performed in this study. The first one consists in evaluating short sequences of texts, so the the architectures are trained and the gender of the subject is computed based on the average classification scores of all short texts from the same subject. Note that for PAN17 corpus, each tweet is a short text, while for call-center transliterations each conversation is divided into chunks with 60 words, similar to the proposed in [10. The second experiment consists in evaluating long assessment of texts. In this case, the complete text data from the subjects is entered to the network at the same time. For the PAN17 corpus all Tweets are concatenated, and for the call-center conversations we consider the complete transliteration of each conversation. This strategy is only evaluated using the CNN-based approach because longer segments produced vanishing gradient problems in the Bi-LSTM network.

Experiments with PAN17: The results obtained for the PAN17 corpus (informal language) are shown in Table 1 for both approaches, short and long texts evaluation. Bi-LSTM and CNN networks are considered. The best results are obtained using the strategy with long texts in the CNN. There is an improvement of up to $4 \%$ in the accuracy per subject with respect to the accuracy obtained with short texts. The improvement in the F1-score is around $2 \%$.

Experiments with Call-Center Conversations: The results observed for the call center conversations (formal language) are shown in Table2. The results include those obtained with and without applying transfer learning. The results also include the ones obtained using short and long texts. The results for this corpus are obtained following a 10-fold cross-validation strategy due to the small size of the corpus. The highest accuracy is obtained here also with the long texts, similar to the results obtained with the PAN17 corpus. In addition, note that the accuracy improves in up to $20 \%$ when the transfer learning strategy is applied 
Table 1. Results of the gender classification in the PAN-CLEF 2017 database.

\begin{tabular}{lccc} 
& \multicolumn{2}{c}{ Short texts } & Long texts \\
& $B i-L S T M$ & $C N N$ & $C N N$ \\
\hline Accuracy per Tweet & 60.5 & 61.1 & - \\
Accuracy per subject & 71.3 & 71.9 & $\mathbf{7 5 . 9}$ \\
Precision & 68.6 & 81.1 & $\mathbf{7 5 . 6}$ \\
Recall & 72.0 & 68.0 & $\mathbf{7 6 . 1}$ \\
F1-Score & 70.8 & 73.9 & $\mathbf{7 5 . 8}$ \\
\hline
\end{tabular}

with respect to the accuracy obtained without using the pre-trained models. Note also that models using transfer learning show a smaller standard deviation which likely indicates that these methods are more stable.

Table 2. Results of the gender classification in the call-center conversations data. TL: transfer learning.

\begin{tabular}{lcccc|cc} 
& \multicolumn{4}{c}{ Short texts } & \multicolumn{2}{c}{ Long texts } \\
& Bi-LSTM & Bi-LSTM & CNN & CNN & CNN & CNN \\
& Without TL & With TL & Without TL & With TL & Without TL & With TL \\
\hline Accuracy per text & $52.7 \pm 6.43$ & $51.6 \pm 5.07$ & $57.9 \pm 9.20$ & $58.3 \pm 6.48$ & - & - \\
Accuracy per subject & $54.2 \pm 10.1$ & $56.4 \pm 12.1$ & $65.9 \pm 12.7$ & $62.9 \pm 14.9$ & $55.9 \pm 11.9$ & $\mathbf{7 5 . 0} \pm \mathbf{6 . 1 8}$ \\
Precision & $65.3 \pm 29.2$ & $55.0 \pm 13.8$ & $52.0 \pm 22.2$ & $61.1 \pm 17.9$ & $54.6 \pm 25.4$ & $\mathbf{7 7 . 2} \pm \mathbf{8 . 1 2}$ \\
Recall & $53.3 \pm 22.7$ & $56.7 \pm 13.2$ & $70.4 \pm 17.2$ & $64.6 \pm 15.2$ & $54.9 \pm 34.8$ & $\mathbf{7 2 . 1} \pm \mathbf{1 0 . 4}$ \\
F1-Score & $55.2 \pm 19.8$ & $55.2 \pm 11.9$ & $57.8 \pm 19.9$ & $61.1 \pm 15.7$ & $48.5 \pm 24.0$ & $\mathbf{7 3 . 8} \pm \mathbf{6 . 0 6}$ \\
\hline
\end{tabular}

\section{Conclusions}

We proposed a methodology for automatic gender classification based on formal texts such as those available in social media posts, and based on formal texts collected in call center conversations. Different deep learning models are evaluated including one on Bi-LSTMs, another one based on CNNs and a transfer learning approach, which is pre-trained with data collected from social networks. The transfer learning method is fine-tuned to improve the accuracy of the model designed for text classification in formal languages. The results indicate that it is possible to classify the gender of a person based on his/her written texts with accuracies of about $75 \%$ in informal and formal language scenarios. The use of a transfer learning strategy improved the accuracy in scenarios where it is more difficult to collect data like in call-center conversations, indicating that this strategy is suitable for companies or sectors where it is not possible to create large datasets from scratch. The models using transfer learning are also more stable and generalize better than others where the neural networks are trained from scratch. This is very positive since it is possible to benefit from the large amounts of text data that are available in other domains like the social networks. The proposed approaches can be extended to other applications related 
to demographic information retrieval such as age recognition, geographic location, personality of the subjects, and others, which would allow the building of more complete and specific author/customer profiles.

\section{Acknowledgments}

This work was funded by the company Pratech Group S.A.S and the University of Antioquia, grant \# PI2019-24110. We would like to thank the Natural Language Engineering Laboratory of the Universidad Politécnica de Valencia for providing access to one of the the databases used in this work.

\section{References}

1. Akhtyamova, L., Cardiff, J., Ignatov, A.: Twitter author profiling using word embeddings and logistic regression. In: Conference and Labs of the Evaluation Forum (CLEF) (Working Notes) (2017)

2. Arras, L., Montavon, G., Müller, K.R., Samek, W.: Explaining recurrent neural network predictions in sentiment analysis. arXiv:1706.07206 (2017)

3. Basile, A., et al.: N-gram: New groningen author-profiling model. arXiv:1707.03764 (2017)

4. Dogan, O., Oztaysi, B.: Gender prediction from classified indoor customer paths by fuzzy c-medoids clustering. In: International Conference on Intelligent and Fuzzy Systems (INFUS). pp. 160-169. Springer (2019)

5. Fernandez-Lanvin, D., de Andres-Suarez, J., Gonzalez-Rodriguez, M., ParienteMartinez, B.: The dimension of age and gender as user model demographic factors for automatic personalization in e-commerce sites. Computer Standards \& Interfaces 59, 1-9 (2018)

6. González Bermúdez, M.: An analysis of twitter corpora and the differences between formal and colloquial tweets. In: Proceedings of the Tweet Translation Workshop 2015. pp. 1-7. CEUR-WS. org (2015)

7. Gu, J., Yu, Z.: Data annealing for informal language understanding tasks. arXiv:2004.13833 (2020)

8. Hirt, R., Kühl, N., Satzger, G.: Cognitive computing for customer profiling: meta classification for gender prediction. Electronic Markets 29(1), 93-106 (2019)

9. Hsieh, F., Dias, R., Paraboni, I.: Author profiling from facebook corpora. In: Proceedings of the Eleventh International Conference on Language Resources and Evaluation (LREC 2018) (2018)

10. Kodiyan, D., et al.: Author profiling with bidirectional rnns using attention with grus. In: Conference and Labs of the Evaluation Forum (CLEF). vol. 1866. RWTH Aachen (2017)

11. Li, W., Dickinson, M.: Gender prediction for chinese social media data. In: Conference Recent Advances in Natural Language Processing (RANLP). pp. 438-445 (2017)

12. Markov, I., Gómez-Adorno, H., Sidorov, G.: Language-and subtask-dependent feature selection and classifier parameter tuning for author profiling. In: Notebook for PAN at Conference and Labs of the Evaluation Forum (CLEF) (2017) 
13. Martinc, M., Skrjanec, I., Zupan, K., Pollak, S.: Pan 2017: Author profiling-gender and language variety prediction. In: Conference and Labs of the Evaluation Forum (CLEF) (Working Notes) (2017)

14. Minaee, S., Azimi, E., Abdolrashidi, A.: Deep-sentiment: Sentiment analysis using ensemble of cnn and bi-lstm models. arXiv:1904.04206 (2019)

15. Otter, D.W., et al.: A survey of the usages of deep learning for natural language processing. IEEE Trans. on Neural Networks and Learning Systems (TNNLS) (2020)

16. Rangel, F., Rosso, P., Potthast, M., Stein, B.: Overview of the 5th author profiling task at pan 2017: Gender and language variety identification in twitter. Working notes papers of the Conference and Labs of the Evaluation Forum (CLEF) pp. 1613-0073 (2017)

17. Torfi, A., Shirvani, R.A., Keneshloo, Y., Tavvaf, N., Fox, E.A.: Natural language processing advancements by deep learning: A survey. arXiv:2003.01200 (2020)

18. Trofimovich, J.: Comparison of neural network architectures for sentiment analysis of russian tweets. In: Computational Linguistics and Intellectual Technologies: Proceedings of the International Conference "Dialogue". pp. 50-59 (2016) 